\title{
Design and Synthesis of Novel Inhibitor against Ser121 and Val122 Mutations in P53 Cancer Gene
}

\author{
Muhammad Hamza", Azhar Mahmood, Sajid Khan, Muhammad Rizwan, Anum Munir \\ Department of Bioinformatics, Government Post Graduate College Mandian, Pakistan
}

Received August 19, 2019; Revised September 25, 2019; Accepted September 27, 2019

Copyright $\mathrm{C} 2019$ by authors, all rights reserved. Authors agree that this article remains permanently open access under the terms of the Creative Commons Attribution License 4.0 International License

\begin{abstract}
The p53 is also known as a tumor suppressor gene, involved in a variety of cellular processes and signaling pathways. p53 mutations are involved in almost all kinds of cancers, and several treatments are available for p53 mutations but have a number of limitations. Still, there is a need for better drugs. Computational methods are emerging and beneficial tools to guide and interpret experiments to fasten the drug design process. This study was undertaken to design a drug that targets p53 Ser121 and Val122 mutations. The compound was identified through virtual screening and several drug-like filters were applied. The identified compound is considered to be non-toxic in nature. ADMET properties and pharmacokinetics of the compound also describe the effectiveness of the compound. The results of this study, suggest that this compound can be used to treat p53 mutations and the compound is synthesized successfully in the lab to determine its adequacy and efficacy. Bis-(4-chlorophenyl)methyl-BLAH compound can be used as a strong inhibitor of p53 Ser 121 and Val 122 mutations.
\end{abstract}

Keywords P53, Docking, Mutations, Synthesized, Medicine, Cancer

\section{Introduction}

The p53 (TP53), a tumor suppressor gene was discovered in 1979, as a protein responsible for tumorigenesis [1]. p53 was considered to be a model in cancer research [2] and situated on chromosome 17, it is responsible for encoding a nuclear protein which controls cell growth and regulation of the cell in S-phase [3]. The p53 is stated to be the paramount gene that is responsible for cancer progression and formation and universally considered a beneficial protein to the organism. Because its absence has disastrous effects: developmental malformations, deregulated metabolism, genomic instability, cancer, unleashed acute inflammation, etc. [4].

Commonly p53 is called as "Guardian of the Genome," etc. [5], p53 plays its biological role by regulating the transcription of many target genes involved in apoptosis, cell cycle arrest, senescence, DNA repair, and transcription factor [6]. Its transcriptional activity enormously increased in cells suffering many types of stresses such as DNA damage, oxidative stress, ribosomal stress or telomere erosion, due to the mutual effect of post-transcriptional and transcriptional regulatory processes, later on, phosphorylation, acetylation, ubiquitousness, modulation, simulation, methylation, are the other factors disturbing the p53 activity, stability and localization [1].

The germline mutations in p53 were discovered by the end of the 1990s as the cause of complicated marital cancer syndromes, which opened a quickly growing research area. These mutations lead to the malignant transformations of target cells and organs, resulting in more than half of human cancers harboring p53 mutations, and mutant p53 allele types prejudice humans to the Li-Fraumeni tumor syndrome [7]. This proves the importance of p53 as a key mainstay in the body's integral anticancer resistance mechanisms $[8,9]$. Wild-type p53 is triggered in feedback to cellular stresses, such as hypoxia, DNA damage and oncogene activation. Missense mutations are the most common mutations of the p53 DNA-binding domain, which produce a full-length protein that is unable to bind to DNA and remains nonfunctional [10]. The most commonly occurring cancers due to p53 mutations involve; cervical cancer, testicular cancer, malignant melanoma, ovarian, neck, head, lungs, larynx, primary leukemia, sarcoma, colorectal and esophageal cancer [11].

Several treatment measures are available for p53 mutations, however, computational methods are emerging as beneficial tools to guide and interpret experiments to fasten the drug design process. There are two main types of computer-aided drug design (CADD); Structure-based drug design and the ligand-based drug design [12]. CADD has been made an essential part of drug discovery and 
forming efforts in the field of biotechnology and pharmaceutical industry [13]. Similarly, another concept of "Pharmacophore" was introduced by Pail Ehrlich at the end of 19th century, meaning that biological activity of a compound depends upon the groups that make a molecule [14]. In modern computational chemistry, Pharmacophore in computational chemistry is used to describe the same biological activity by one or many molecules having some essential features [12].

As the p53 is a major contributor to the number of cancers in the human body, therefore, this study is an attempt to determine the most effective drug compound against P53 Se121 and Val122 mutations through virtual screening method.

\section{Materials and Methods}

The detailed procedure of methodology is shown in Figure 1.

\subsection{Retrieval of Gene Information}

The information about the p53 gene was obtained through literature and its estimated ratio of disease involvement were collected from GeneCards. GeneCards is an integrative database that provides understandable and user-friendly information about all the human genes. It merges the data from 125 sources, including genomics, transcriptomics, proteomics, genetic, clinical, and functional information. GeneCards can be accessed freely [15].

\subsection{Virtual Screening of Compounds Against P53 Gene}

Virtual screening is done by computer-based methods for the discovery of novel ligands based on biological structures [16]. Keeping this in mind, virtual screening of compounds that can be used to treat $\mathrm{p} 53$ mutations was performed through the ZINC database. The ZINC is a database for commercially available drug compounds, the biologists, chemists, and researcher from the biotech, academia, and pharmaceutical industries especially used this database for virtual screening [17].

\subsection{Mining of Compounds on Lipinski, Veber, Ghose, Muegge and Egan Rule}

For mining of the dataset of p53, the Lipinski, Veber, Ghose, Muegge, and Egan rules were used: Lipinski rule states that a drug like compound must have molecular weight $(\mathrm{Mw})$ less than 500 Daltons (D), LogP less than 5, $\mathrm{H}$ - bond donors (HBD) less than or equal to 5, H-bond acceptors (HBA) less than or equal to 10 [18], According to Veber and Ghose Mw must be in range of 160 to $480, \log \mathrm{P}$ between -0.4 - 5.6 [19], Muegge rule states that mw should be $200-600, \log$ P between -2 to 5, HBA less than $10, \mathrm{HBD}$ less than 5, No of Rotatable bonds less than or equal to 15 and Egan Rule says that LOGP must be less than 5.88 [20].

\subsection{Selection of Compounds Fulfilling all Rules}

After applying all the Lipinski, Veber, Ghose, Muegge and Egan Rules on the virtual screened data, several compounds were selected fulfilling all the rules.

\subsection{Toxicity Analysis}

The protox-II webserver was used for toxicity verification of compounds as well as to determine their carcinogenic, immunogenic, hepatotoxic, and cytotoxic nature. ProTox web server, have both molecular and chemical target information. Toxicity classes in ProTox server are defined according to the globally harmonized system of classification of labeling of chemicals (GHS), class I and II is fatal, class III is toxic, class IV is harmful, and class V may be harmful while class VI is non-toxic in nature.

ProTox-II is a freely available server for toxicity prediction of compounds [21]. LD50 is an approach that yields an in-silico drug compound for our particular proteins.

\subsection{Pharmacophore Generation and Health Effect Prediction}

LigandScout was used for the 3D Pharmacophore Generation [22]. LigandScout is used for the detection of a significant interaction in ligand-protein complex [23]. Health effects were predicted by ACD/I-Lab. Health effects are checked in order to identify the minimal probabilities of side effects if the side effect of the compound is high, the compound will be more toxic in nature [24].

\subsection{Identification of Mutated Pocket of P53 Gene}

The mutated $\mathrm{p} 53$ protein was downloaded from the PDB database after applying filters, the mutated pocket of P53 gene was identified through PockDrug server [25]. Protein pockets are basically identified to determine the ability of the drug with high affinity.

\subsection{Molecular Docking of Potent Compound}

Molecular docking is a mostly used method in drug design because of its accurate prediction and confirmation of ligand with the target binding site [26]. The molecular docking of the selected lead compound was performed with the mutated pocket of P53 protein through auto dock tool, binding energy, binding affinity, and inhibition constants were determined. 


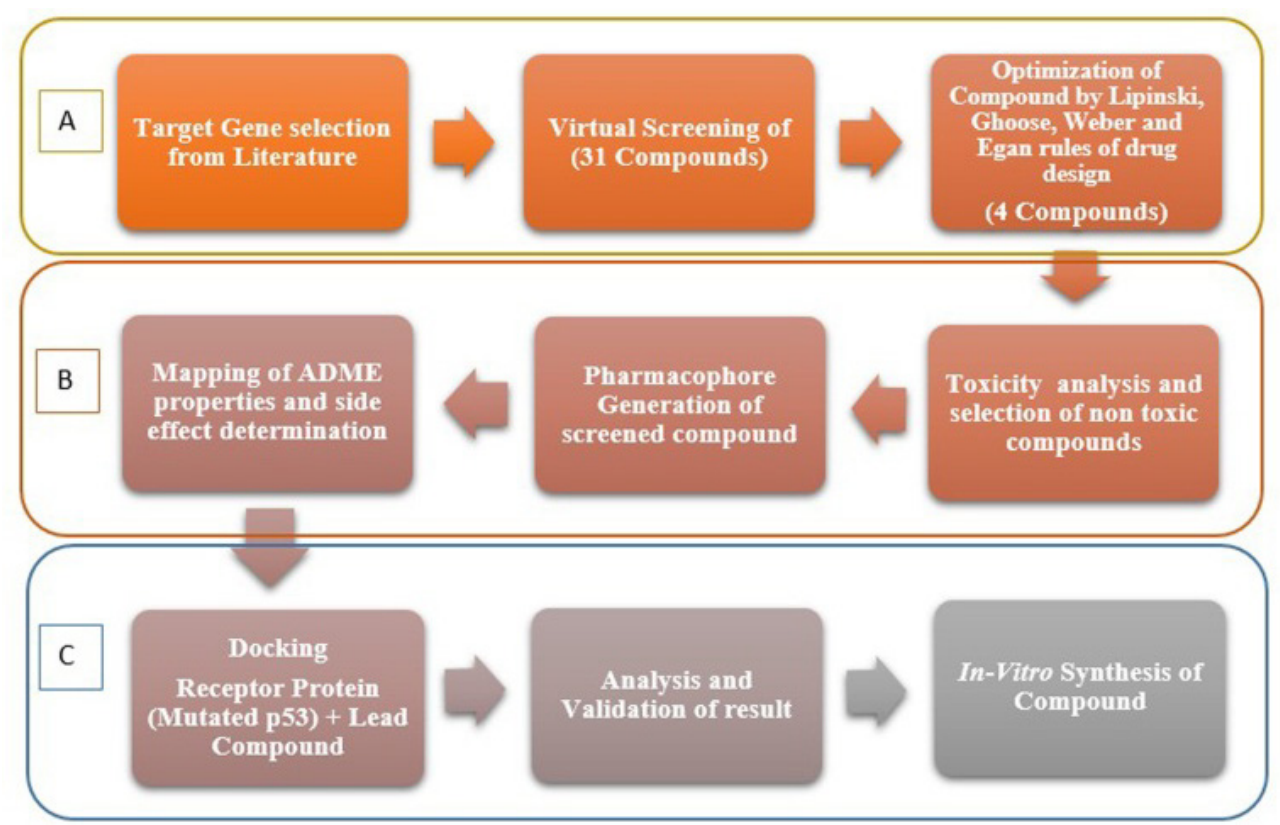

Figure 1. Detailed flow chart of Methodology used for the research work; A) Target optimization, B) Screening, C) Analysis and validation

\subsection{Synthesis of Bis-(4-chlorophenyl)methyl-BLAH}

In the presences of certain catalysts, Bis-(4-chlorophenyl)methyl-BLAH is synthesized In-Vitro by adding methyl iodide and bis-4-chlorophenyl inappropriate chemical settings.

\subsection{Cytotoxicity and Anti-cancer Activity}

The cytotoxic assays were performed on the cancer cell line Promyeloblast leukemia and Haematopoietic and lymphoid tissue in Leukemia tumor cell having mutation in P53 and anticancer activity was determined using CLCPred.

\section{Results}

A total of 31 compounds were retrieved from the ZINC database after virtual screening and only 4 of them fulfill the drug-likeness rules (Lipinski, Veber, Ghose, Muegge and Egan Rule). In the toxicity prediction of 4 compounds, 3 compounds were lying in toxicity class 4 , and only one compound was lying in $5^{\text {th }}$ class; nontoxic in nature having ZINC ID ZINC45319528, LogP 2.03, HBD 0, HBA 5, Mol-Weight 462.36, Rotatable Bonds 2 and toxicity class $\mathrm{V}$, which confirms the effectiveness of compound and its drug-like nature. The pharmacophore model of the ligand was also generated to confirm its drug-like features (figure 2).

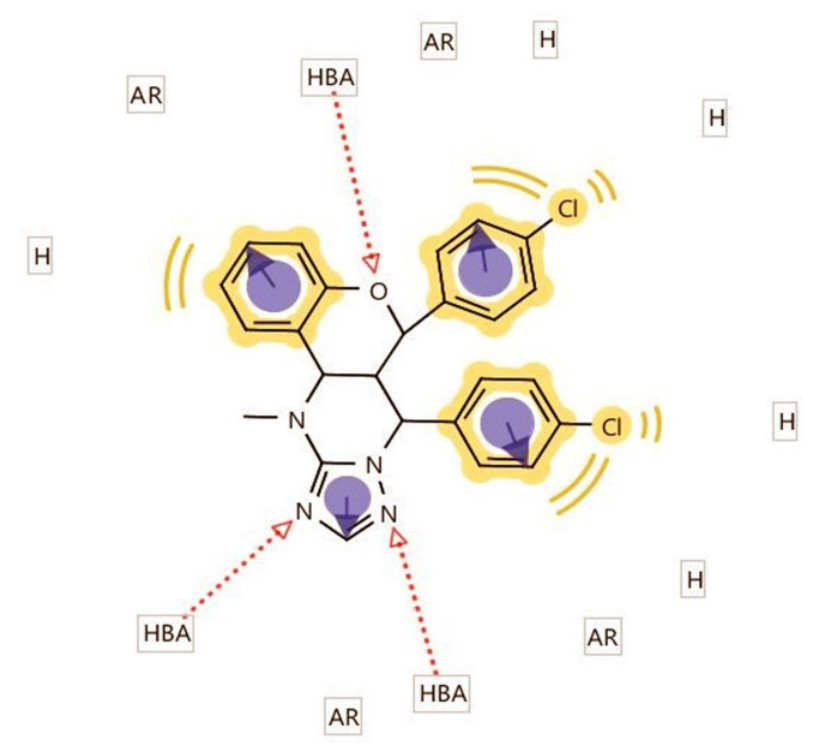

Figure 2. The pharmacophore model of the selected ligand, HBA represents Hydrogen bond acceptor, $\mathrm{H}$ represents the hydrophobic region and $\mathrm{AR}$ represents Aromatic ring.

The pharmacophore model of the selected ligand shows three important features HBA, hydrophobic region $(\mathrm{H})$, and aromatic rings (AR). The drug-likeness characteristics are identified to determine whether a lead compound is satisfying the drug-like properties [27]. The basic physio-chemical properties of the ligand are shown in Table 1. 
Table 1. Physiochemical properties of ligand compound

\begin{tabular}{|c|c|c|c|c|c|c|}
\hline Molecular Formula & Ligand Compound & Lipinski Ro5 & Ghose Rule & Vebers Rule & Egans Rule & Muegge Rule \\
\hline Mol-Weight & 462.36 & $<=500$ & $160-480$ & $<=500$ & NA & NA \\
\hline LogP & 2.03 & $0-5$ & $-0.4-5.6$ & NA & $0-8$ & NA \\
\hline H-Bond donor & 0 & $<=5$ & NA & $<=12$ & NA & $<6$ \\
\hline H-Bond acceptor & 5 & $<=10$ & NA & $<=12$ & NA & $<6$ \\
\hline Rotatable Bonds & 2 & $<=10$ & NA & $<=10$ & NA & NA \\
\hline TPSA & $42.95 \AA^{2}$ & NA & NA & $<=140$ & $74-92$ & $66-90$ \\
\hline Molar Refractivity & 130.23 & NA & $40-130$ & NA & NA & NA \\
\hline
\end{tabular}

Table 1 shows the physicochemical properties of ligand compounds fulfilling Lipinski, Ghose, Veber, Egans and Muegge rule, it is also observed that the ligand compound fulfills all the properties of a drug-like compound The pharmacokinetic properties of the selected ligand were also determined through Swiss ADME tool for further validation. These properties are shown in table 2.

Table 2. The Water solubility and pharmacokinetics of Ligand compound by Swiss ADME server

\begin{tabular}{|c|c|}
\hline \multicolumn{2}{|c|}{ Water solubility } \\
\hline Log S (ESOL) & -6.61 \\
\hline Solubility & $1.14 \mathrm{e}-04 \mathrm{mg} / \mathrm{ml} ; 2.46 \mathrm{e}-07 \mathrm{~mol} / 1$ \\
\hline Class & Poorly soluble \\
\hline $\log$ S (Ali) & -6.22 \\
\hline Solubilty & $2.77 \mathrm{r}-04 \mathrm{mg} / \mathrm{ml} ; 5.99 \mathrm{e}-07 \mathrm{~mol} / 1$ \\
\hline Class & Poorly soluble \\
\hline Log S (SILICOS- IT) & -9.26 \\
\hline Solubility & $2.55 \mathrm{e}-07 \mathrm{mg} / \mathrm{ml}: 5.52 \mathrm{e}-10 \mathrm{~mol} / 1$ \\
\hline Class & $\begin{array}{l}\text { Poorly Soluble } \\
\end{array}$ \\
\hline \multicolumn{2}{|c|}{ Pharmacokinetics } \\
\hline GI absorption & High \\
\hline BBB permanent & Yes \\
\hline P-gp substrate & Yes \\
\hline CYP1A2 inhibitor & No \\
\hline CYP2C19 inhibitor & Yes \\
\hline CYP2C9 & No \\
\hline CYP2D6 inhibitor & No \\
\hline CYP3A4 inhibitor & No \\
\hline $\log \mathrm{Kp}$ & $-5.17 \mathrm{~cm} / \mathrm{s}$ \\
\hline Synthetic accessibility & 4.80 \\
\hline
\end{tabular}

From the table 2, it is observed that GI (Gastrointestinal) Absorption of this compound is high which means that this drug will be more effectively bind to the target and will easily be absorbed in the body, drugs having higher absorption rates are more effective in nature. CYP is the metabolic enzymes involve in the formation and breakdown of various molecule and chemical within the cell. The compound is non-inhibitor of all CYP enzymes which shows the effectiveness of the compound. The ADMET properties were predicted from the PKCSM are shown in table 3.

Table 3 shows the ADMET properties of the ligand compound checked by PKCSM server. The table illustrates that the compound does not produce HAPs toxicity, No Hepatotoxicity (harmful to the liver caused by a medication, chemical, or herbal) is produce and no skin sensation occur. The use of ADMET properties is becoming more vital in the drug discovery, assortment, development and promotion processes [28]. The health effects of the selected ligand were also determined to know

the probability of its side effects on the major areas and important organs of the body. The probability of health effects is given in table 4 .

Table 3. ADMET properties of Ligand Compound by PKCSM server

\begin{tabular}{|c|c|c|}
\hline Model Name & $\begin{array}{l}\text { Predicted } \\
\text { Value }\end{array}$ & Unit \\
\hline \multicolumn{3}{|c|}{ Absorption } \\
\hline Water solubility & -5.336 & $(\log \mathrm{mol} / \mathrm{L})$ \\
\hline Caco2 permeability & 1.032 & $\begin{array}{c}\text { (log Papp in } \\
10^{-6} \mathrm{~cm} / \mathrm{s} \text { ) }\end{array}$ \\
\hline Intestinal absorption (human) & 98.818 & (\% Absorbed) \\
\hline Skin Permeability & -2.734 & $(\log \mathrm{Kp})$ \\
\hline P-glycoprotein substrate & No & Categorical \\
\hline P-glycoprotein I inhibitor & Yes & Categorical \\
\hline P-glycoprotein II inhibitor & Yes & Categorical \\
\hline \multicolumn{3}{|c|}{ Distribution } \\
\hline VDss (human) & 0.08 & $(\log \mathrm{L} / \mathrm{kg})$ \\
\hline Fraction unbound (human) & 0.238 & $(\mathrm{Fu})$ \\
\hline BBB permeability & 0.145 & $(\log \mathrm{BB})$ \\
\hline CNS permeability & -1.157 & $(\log$ PS) \\
\hline \multicolumn{3}{|c|}{ Metabolism } \\
\hline CYP2D6 substrate & No & Categorical \\
\hline CYP3A4 substrate & Yes & Categorical \\
\hline CYP1A2 inhibitor & Yes & Categorical \\
\hline CYP2C19 inhibitor & Yes & Categorical \\
\hline CYP2C9 inhibitor & Yes & Categorical \\
\hline CYP2D6 inhibitor & No & Categorical \\
\hline CYP3A4 inhibitor & No & Categorical \\
\hline \multicolumn{3}{|c|}{ Excretion } \\
\hline Total Clearance & 0.139 & $(\log \mathrm{ml} / \mathrm{min} / \mathrm{kg})$ \\
\hline Renal OCT2 substrate & No & Categorical \\
\hline \multicolumn{3}{|c|}{$\begin{array}{ll}\text { Toxicity } \\
\end{array}$} \\
\hline AMES toxicity & No & Categorical \\
\hline Max. tolerated dose (human) & 0.406 & $\begin{array}{c}(\log \\
\mathrm{mg} / \mathrm{kg} / \text { day })\end{array}$ \\
\hline hERG I inhibitor & No & Categorical \\
\hline hERG II inhibitor & Yes & Categorical \\
\hline $\begin{array}{c}\text { Oral Rat Acute Toxicity } \\
\text { (LD50) }\end{array}$ & 2.868 & $(\mathrm{~mol} / \mathrm{kg})$ \\
\hline $\begin{array}{c}\text { Oral Rat Chronic Toxicity } \\
\text { (LOAEL) }\end{array}$ & 0.274 & $\begin{array}{c}\text { (log } \\
\mathrm{mg} / \mathrm{kg} \text { _bw/day) }\end{array}$ \\
\hline Hepatotoxicity & No & Categorical \\
\hline Skin Sensitisation & No & Categorical \\
\hline T.Pyriformis toxicity & 0.285 & $(\log u g / L)$ \\
\hline Minnow toxicity & -1.054 & $(\log \mathrm{mM})$ \\
\hline
\end{tabular}


Table 4. Health effects of the compound on Blood, Cardiovascular system, gastrointestinal system, kidney, liver, and lungs

\begin{tabular}{|c|c|}
\hline \multicolumn{2}{|c|}{ Health Effects } \\
\hline Blood & 0.9 \\
\hline Cardiovascular system & 0.92 \\
\hline Gastrointestinal system & 0.99 \\
\hline Kidney & 0.91 \\
\hline Liver & 0.59 \\
\hline Lungs & 0.62 \\
\hline
\end{tabular}

Table 4 represents the probability of effect on Blood, Cardiovascular system, gastrointestinal system, Kidney, Liver and Lungs, probability of effects on each organ is less than one which means the compound is effective in nature and nontoxic with least side effects. The mutated protein was downloaded from RCSB-PDB having ID 4MZR with 2 mutations Ser 121 and Val 122. The active mutant pockets of the protein were determined through Pock Drug server. About 34 pockets were predicted having different probability values, among these pockets, P29 was selected because of its probability value 1.0, standard deviation 0 which means there is no error. Pocket P29 is then selected for docking, it has drug probability value 1.0 along with aromatic residue 0.1 and hydrophobic Kyte 3.16, polar residue proportion 0.07 and volume Hull 344.06 respectively. This pocket was selected for Molecular docking with the ligand compound. The active pocket of mutated P53 protein is shown in figure 3 and the result of docking are shown in figure 4.

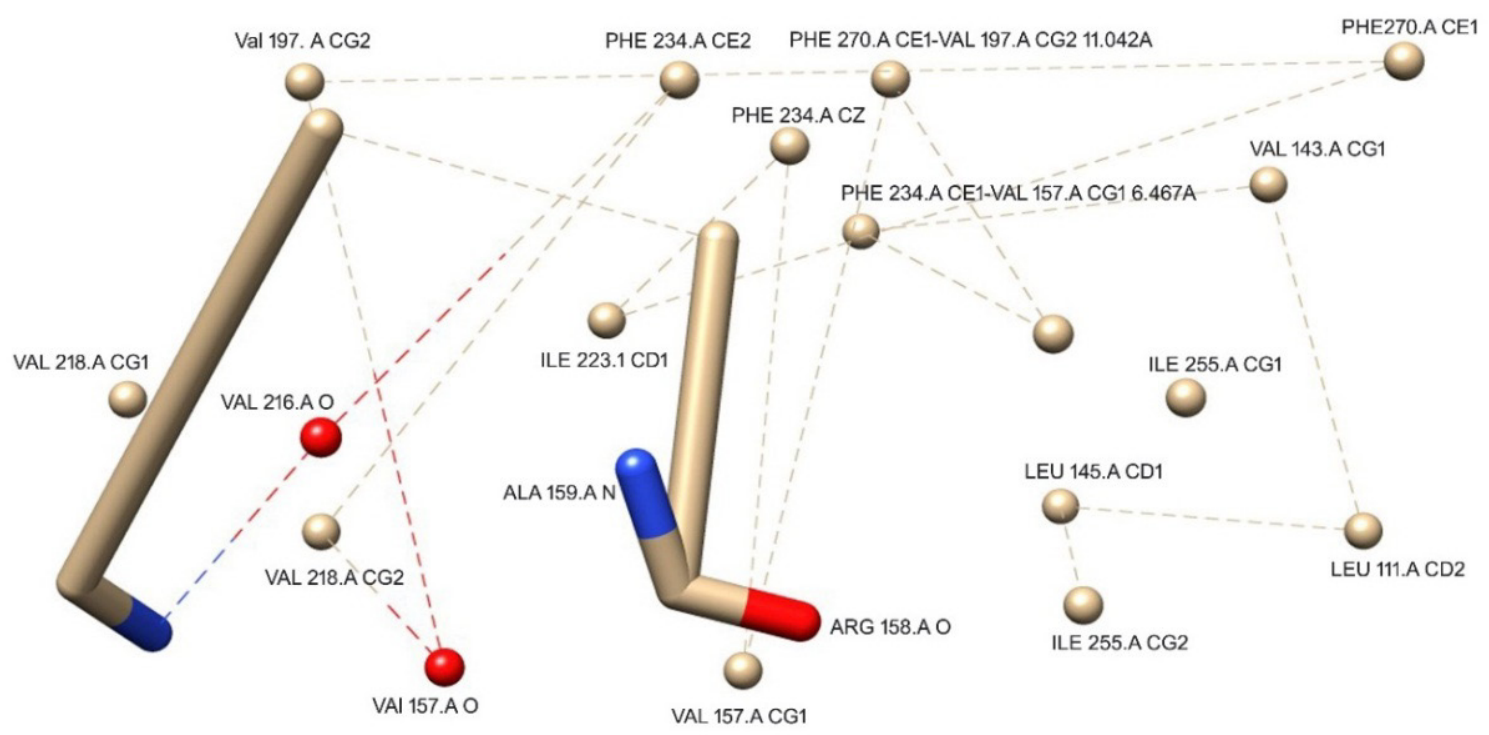

Figure 3. Identification of mutant pocket by PockDrug server.
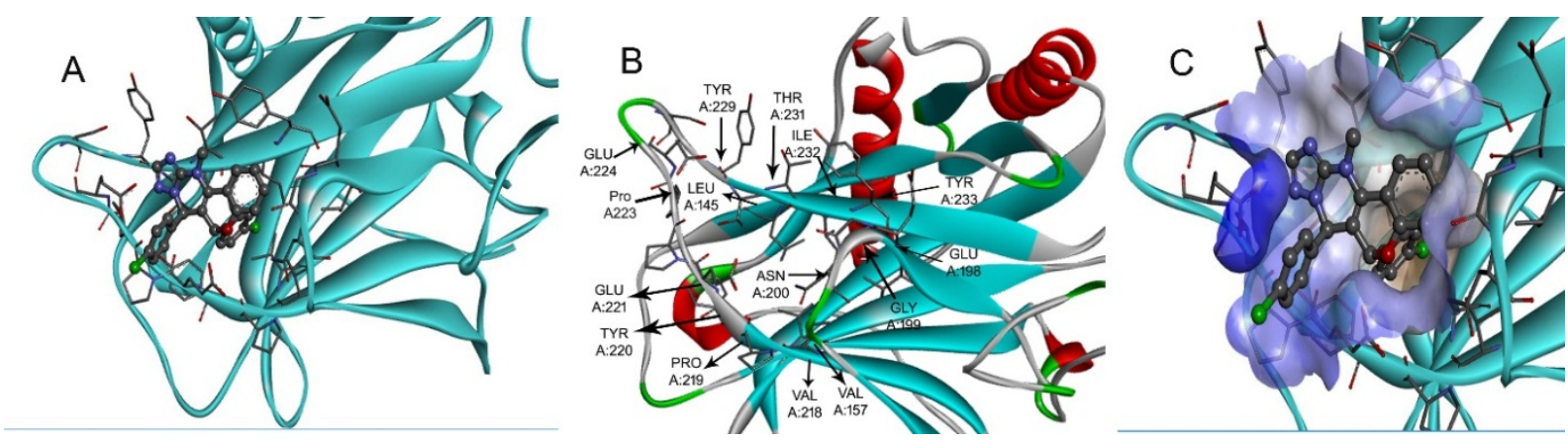

Figure 4. (A-C). A) Receptor Ligand Complex, B) 2D mapping of interacting residues, C) Best Pose of the Lead compound and Hydrophobic Surface of target Protein

Figure 4 is the 3D complex of Protein after docking, the ligand perfectly binds to the active pocket site of protein 4MZR, which describe the effectiveness of the drug compound. The hydrophobic surface of the drug compound was determined, if the drug is hydrophobic in nature, the drug compound will reach to the target site. This drug compound is hydrophobic in nature and will bind to the target site accurately. Figure 5 describe the interaction of ligand compound with amino acid residues of the mutated pocket. 


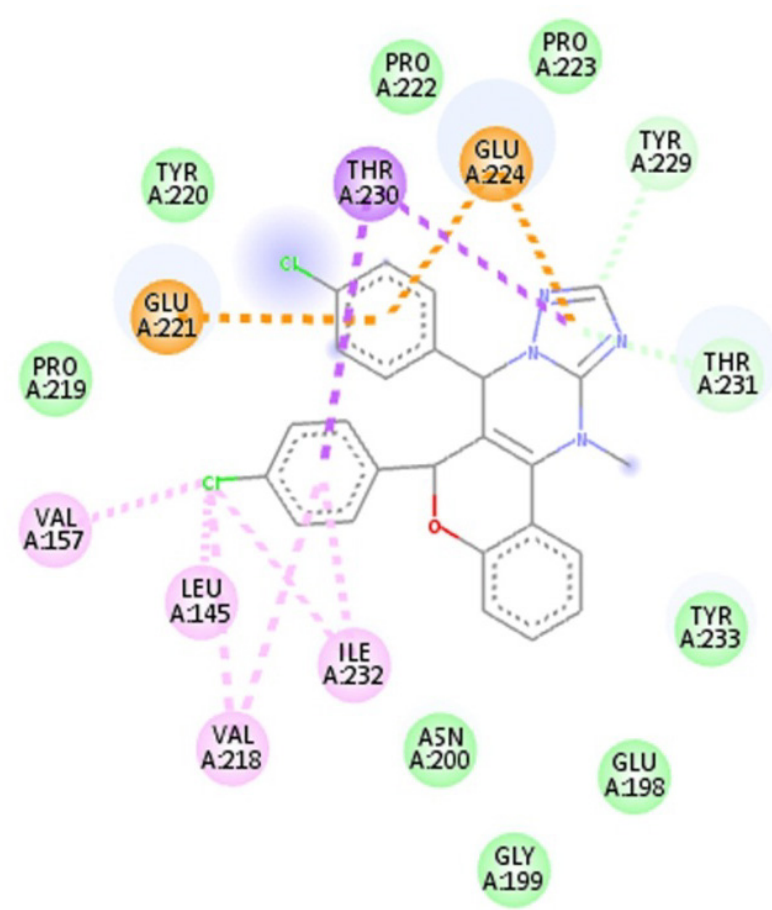

Figure 5. 2D Interaction of this Drug Compound.

From figure 5, it is observed that the compound formed Alkyl and Pi-Alkyl bonds with VAL, LEU and ILE, Pi-Anion bond with GLU, and sigma bond with THR amino acid. In the visualization of $2 \mathrm{D}$ interaction of this protein complex, no bumps were found in the docked complex of protein. Bumps are usually produced due to the collision of two molecules with each other, allowable number of bumps are mostly 3 in molecular docking.

From the aforementioned results, it is suggested that this compound is an effective inhibitor of p53 mutations. The compound Bis-(4-chlorophenyl) methyl-BLAH was synthesized In-Vitro by following a significant procedure.

50 grams of red phosphorus and 180 grams of methanol were taken in a round bottom flask with a reflux condenser. Then gradually add 500 grams of iodine to the reaction mixture. A considerable heat starts to occur and the reaction flask was left attached to the condenser full night when the iodine had been added. The next day methyl iodide crude was distilled, in a separating funnel, the distillate was washed using sodium hydroxide dilute solution in order to remove hydriodic acid and iodine. Methyl iodide was separated and dried up with calcium chloride, and end with distillation for purification.

Bis-(4-chlorophenyl)methyl-BLAH was prepared by reduction of 4-chlorocinnamic acid., For hydrogenations, the readily dehydrogenated tetralin was used, as a substitute of molecular hydrogen and combine Methyl Iodide with it.

$5 \mathrm{~g}$ of 4-chlorocinnamic acid solution was added in 180 $\mathrm{ml}$ tetralin having $1 \mathrm{~g}$ of palladium black was heated for 1.5 hours at the boiling point in a flask in an air condenser. On the completion of the reaction, the catalyst was cleaned off.
Sodium carbonate solution was used to remove the unreacted 4-chlorocinnamic acid and 3-(4-chlorophenyl) from the filtrate, then ether was used to remove adhering tetralin from the alkaline solution, then Hydrochloric acid was added to the alkaline solution to become acidified, and the Bis-(4-chlorophenyl) acid that separates was taken up in ether. On evaporation, melting point $123^{\circ} \mathrm{C}$ was obtained for pure Bis-(4-chlorophenyl) acid. The tetralin in the experiment was purified with dilute hydrochloric acid by extraction, washing, and calcium chloride for drying and then distilled (b.p $207^{\circ} \mathrm{C}$ )

Purified 180 grams of the Bis-(4-chlorophenyl) acid was mixed with 50 grams of methyl iodide in a flask under moderate heating in the presence of a catalyst, potassium up to 30 minutes and kept in air condenser. The yield pure Bis-(4-chlorophenyl)methyl-BLAH was distilled approximately the theoretical amount.

The in-vitro cytotoxic assays were performed using SRB (Sulphorhodamine B) assay. The Probability of cytotoxic activity in tumor cell line is greater than $\mathrm{P}=0.612$ which explains the cytotoxicity is $60 \%$ tumor cell line. The cytotoxicity is checked in cancer cell line of Promyeloblast leukemia and Haematopoietic and lymphoid tissue in Leukemia tumor cell having mutation in P53.The anticancer activity of the compound was found to be very high in several cancers including Haematopoietic and lymphoid tissue, Lungs, Liver, Ovary, Oesophagus, Skin, Breast, Bone etc.

\section{Discussion}

In this work, the screening technique was applied on compounds for screening best compound to be used against $\mathrm{p} 53$. As the $\mathrm{p} 53$ is the major contributor to human cancers $[29,30]$. The lead compound was identified through virtual screening, the health effect of the drug compound was determined which confirmed it as non-toxic in nature for healthy tissues. The active pocket site of the mutated p53 protein was identified by the PockDrug server as shown in figure 3 and the drug compound was docked on the selected pocket, Wolber et al., [31], in their study, identified a low weight compound, PRIMA-1 that induces apoptosis in human cancer cells. PRIMA-1 was selected on the base of low molecular weight; no side effects of the compound were examined for PRIMA-1. Here in this study, the compound was screened on the base of different rules as shown in table 1 , not only different rules were applied but also, number of steps were performed to identify its side effects, toxicity, pharmacokinetic properties, and ADME properties, it was observed that the screened compound was not only non-toxic in nature but as 1 has low molecular weight

STIMA-1 another low MW compound was identified by $\mathrm{Yu}$ et al. [32] to induce the expression of $\mathrm{p} 53$, again the compound was selected only on the bases of low molecular 
weight, no toxicity was checked for the compound and no ADME properties were determined in order to show the effectiveness. Here, not only the toxicity class for the screened compound was identified, but also, the ADME properties were identified and the gastrointestinal absorption was analyzed for the compound which determines the accuracy of the compound which binds to the active binding site of the protein.

Sulfated fucose (marine) was suggested to be the lead drug compound candidate to binds with mutant p53 for the treatment of different types of cancer. The Sulfated fucose has higher molecular weight and a lot of HBD's and HBAs. As the violation of Lipinski rule may lead the compound to cause different side effect ${ }^{33}$. Here the drug compound identified in this scientific study does not violate any drug-like rule, therefore from all the aforementioned results, it is suggested to be used as a strong inhibitor of p53 Ser 121 and Val 122 mutations.

In this research work, we retrieved 31 compounds of $\mathrm{p} 53$ from Zinc database on which screening and mining techniques were applied on the base of Lipinski, Veber, Ghose, Muegge and Egan Rule to check the physiochemical properties of the compound, also toxicity of the compound, health effects, pharmacokinetics, and ADMET properties are check in order to determine the perfectness of the compound. Only one compound fulfills all the rules and was nontoxic in nature. Pharmacophore model was generated for a significant interaction. The compound was docked with the mutated protein of $\mathrm{p} 53$, the pocket was identified through PockDrug server. In the molecular docking result, the compound perfectly bound to the active pocket site of the protein. Following the aforementioned results, the compound was synthesized In-Vitro successfully and can be used as a strong inhibitor of p53 Ser 121 and Val 122 mutations.

\section{Conclusions}

In this research work, we retrieved 31 compounds of p53 from Zinc database on which screening and mining techniques were applied on the base of Lipinski, Veber, Ghose, Muegge and Egan Rule to check the physiochemical properties of the compound, also toxicity of the compound, health effects, pharmacokinetics, and ADMET properties are check in order to determine the perfectness of the compound. Only one compound fulfills all the rules and was nontoxic in nature. Pharmacophore model was generated for a significant interaction. The compound was docked with the mutated protein of $\mathrm{p} 53$, the pocket was identified through PockDrug server. In the molecular docking result, the compound perfectly bound to the active pocket site of the protein. Cytotoxicity of the compound determines the activity in cell line with the probability value greater than 0.5 , which shows the active probability of the compound in cell line. Following the aforementioned results, the compound was synthesized In-Vitro successfully and can be used as a strong inhibitor of p53 Ser 121 and Val 122 mutations.

\section{Acknowledgements}

None of the authors have any challenging conflict of interest. The authors are grateful to the department of Bioinformatics Govt. Postgraduate College Mandian, Abbottabad and Bioinformatics International Research Club GPGCM, for providing a platform to conduct the research.

\section{REFERENCES}

[1] A. Szybińska, W. Leśniak. P53 Dysfunction in Neurodegenerative Diseases-The Cause or Effect of Pathological Changes? Aging and disease, Vol. 8, No. 4, 506, 2017.

[2] C. C. Harris, Structure and function of the p53 tumor suppressor gene: clues for rational cancer therapeutic strategies, JNCI: Journal of the National Cancer Institute, Vol. 88, No. 20, 1442-1455, 1996.

[3] R. Villuendas. p53 protein expression in lymphomas and reactive lymphoid tissue, The Journal of pathology, Vol. 166, No. 3, 235-241, 1992.

[4] M. J. Duffy, N. C. Synnott, J. Crown. Mutant p53 as a target for cancer treatment, European Journal of Cancer, Vol. 83, 258-265, 2017.

[5] A. V. Gudkov, E. A. Komarova. Pathologies Associated with the p53 Response, Cold Spring Harbor Perspectives in Biology, Vol. 2, No. 7, a001180-a001180, 2010.

[6] A. Parrales, T. Iwakuma. Targeting Oncogenic Mutant path for Cancer Therapy, Frontiers in Oncology, Vol. 5, 2015.

[7] C. A. Brady, L. D. Attardi. p53 at a glance, Journal of Cell Science, Vol. 123, No. 15, 2527-2532, 2010.

[8] M. Oren, V. Rotter. Mutant p53 Gain-of-Function in Cancer, Cold Spring Harbor Perspectives in Biology, Vol. 2, No. 2, a001107-a001107, 2010.

[9] J. A. Royds and B. Iacopetta. p53 and disease: when the guardian angel fails, Cell death and differentiation, Vol. 13, No. 6, 1017, 2006.

[10] A. Willis, E. J. Jung, T. Wakefield, X. Chen. Mutant p53 exerts a dominant negative effect by preventing wild-type p53 from binding to the promoter of its target genes, Oncogene, Vol. 23, No. 13, 2330-2338, 2004.

[11] M. Olivier, M. Hollstein, P. Hainaut. TP53 Mutations in Human Cancers: Origins, Consequences, and Clinical Use, Cold Spring Harbor Perspectives in Biology, Vol. 2, No. 1, a001008-a001008, Jan. 2010.

[12] W. Yu, A. D. MacKerell. Computer-Aided Drug Design Methods, Antibiotics, Vol. 1520, P. Sass, Ed. New York, 
NY: Springer New York, 2017, 85-106.

[13] Q. Gao, L. Yang, Y. Zhu. Pharmacophore based drug design approach as a practical process in drug discovery, Current computer-aided drug design, Vol. 6, No. 1, 37-49, 2010.

[14] T. Kaserer, K. Beck, M. Akram, A. Odermatt, D. Schuster, Pharmacophore Models and Pharmacophore-Based Virtual Screening: Concepts and Applications Exemplified on Hydroxysteroid Dehydrogenases, Molecules, Vol. 20, No. 12, 22799-22832, 2015.

[15] G. Stelzer. The GeneCards Suite: From Gene Data Mining to Disease Genome Sequence Analyses: The GeneCards Suite, Current Protocols in Bioinformatics, A. Bateman, W. R. Pearson, L. D. Stein, G. D. Stormo, and J. R. Yates, Eds. Hoboken, NJ, USA: John Wiley \& Sons, Inc., $1.30 .1-1.30 .33,2016$

[16] B. K. Shoichet. Virtual screening of chemical libraries, Nature, Vol. 432, No. 7019, 862, 2004.

[17] J. J. Irwin, B. K. Shoichet. ZINC- A free database of commercially available compounds for virtual screening, Journal of chemical information and modeling, Vol. 45, No. 1, 177-182, 2005.

[18] D. Lagorce, O. Sperandio, H. Galons, M. A. Miteva, B. O. Villoutreix. FAF-Drugs2: Free ADME/tox filtering tool to assist drug discovery and chemical biology projects, BMC Bioinformatics, Vol. 9, No. 1, 396, 2008.

[19] G. R. Bickerton, G. V. Paolini, J. Besnard, S. Muresan, A. L. Hopkins, Quantifying the chemical beauty of drugs, Nature Chemistry, Vol. 4, No. 2, 90-98, 2012.

[20] D. Lagorce, O. Sperandio, J. B. Baell, M. A. Miteva, B. O. Villoutreix. FAF-Drugs3: a web server for compound property calculation and chemical library design, Nucleic Acids Research, Vol. 43, No. W1, W200-W207, 2015.

[21] P. Banerjee, A. O. Eckert, A. K. Schrey, R. Preissner. ProTox-II: a webserver for the prediction of toxicity of chemicals, Nucleic Acids Research, Vol. 46, No. W1, W257-W263, 2018.

[22] G. Wolber, T. Langer. LigandScout: 3-D Pharmacophores Derived from Protein-Bound Ligands and Their Use as Virtual Screening Filters, Journal of Chemical Information and Modeling, Vol. 45, No. 1, 160-169, 2005.

[23] Z. Chen. Pharmacophore-based virtual screening versus docking-based virtual screening: a benchmark comparison against eight targets, Acta Pharmacologica Sinica, Vol. 30, No. 12, 1694-1708, 2009.

[24] A. Munir, S. Malik, S. Aslam, A. Mehmood, S. Amjad, KA. Malik, M. Younis, AH. Shah, GM. Shah2. MEDICINAL PLANTS ARE EFFECTIVE INHIBITORS OF TYPE I AND II DIABETES, 7, 2018.

[25] HA. Hussein, A. Borrel, C. Geneix, M. Petitjean, L. Regad, AC. Camproux. PockDrug-Server: a new web server for predicting pocket druggability on holo and apo proteins. Nucleic Acids Res, No. 43, W436-442, 2015

[26] L. Ferreira, R. dos Santos, G. Oliva, A. Andricopulo. Molecular Docking and Structure-Based Drug Design Strategies, Molecules, Vol. 20, No. 7, 13384-13421, 2015.

[27] D. Datta. Protein-Ligand Interactions: Docking, Design and
Protein Conformational Change, 353.

[28] M. Tareq Hassan Khan. "Predictions of the ADMET properties of candidate drug molecules utilizing different QSAR/QSPR modelling approaches, Current drug metabolism, Vol. 11, No. 4, 285-295, 2010.

[29] M. J. Duffy, N. C. Synnott, P. M. McGowan, J. Crown, D. O'Connor, W. M. Gallagher, p53 as a target for the treatment of cancer, Cancer Treatment Reviews, Vol. 40, No. 10, 1153-1160, 2014.

[30] D. P. Lane, C. F. Cheok, S. Lain. p53-based Cancer Therapy, Cold Spring Harbor Perspectives in Biology, Vol. 2, No. 9, a001222-a001222, 2010.

[31] V. J. Bykov, Restoration of the tumor suppressor function to mutant p53 by a low-molecular-weight compound, Nature medicine, Vol. 8, No. 3, 282, 2002.

[32] N. Zache. Mutant p53 targeting by the low molecular weight compound STIMA-1, Molecular Oncology, Vol. 2, No. 1, 70-80, 2008.

[33] D. Preamnath, R. A. Asir, J. A. Jebamalar, J. J. V. M. P. Gomez. Insilico docking and interaction analysis of bioactive marine compound (sulfated fucose) against the human mutant p53 protein involved in carcinogenesis, International Journal of Scientific \& Engineering Research, Vol. 3, No. 9, 2012. 